\title{
1 MethodiK der StUdie
}

\subsection{Die Samples}

\section{Die Respondent/inn/en}

Für die vorliegende Studie wurden 65 Afghan/inn/en interviewt, davon waren 27 weiblich und 38 männlich, die zum Zeitpunkt der Erhebung in den Bundesländern Wien, NÖ und OÖ verortet waren. Auf Basis der folgenden Variablen wurde garantiert, eine breite Streuung zu erzielen: Ankunft in Österreich, Alter, ethnische Zugehörigkeit, Bildungsstatus und ausgeübter Beruf zum Erhebungszeitpunkt. So wurde einem möglichen „Bias“ entgegengewirkt. Des Weiteren wurden Personen differenziert nach dem Ankunftsjahr in Österreich angefragt, um vergleichende Analysen je nach der Aufenthaltsdauer durchführen zu können. Hierbei wurden fünf Gruppen gebildet: 70er, 80er, 00er/10er Jahre, 2013/2014 und ab 2015. Personen, die sich im Asylverfahren befanden, wurden nicht befragt, sondern ausschließlich solche mit positivem Asylbescheid oder subsidiärem Schutz.

\section{Die Expert/inn/en}

Insgesamt wurden 13 Expert/inn/en aus den verschiedensten Organisationen interviewt, die sich mit asyl- und subsidiär schutzberechtigten Afghan/inn/en in Österreich beschäftigen. Aufgrund der sehr diversen Aufgabenbereiche der Befragten konnte ein möglichst breites Spektrum an Aspekten der Integration von asyl- und subsidiär schutzberechtigten Afghan/inn/en abgedeckt werden.

Insbesondere Repräsentant/inn/en beratender Organisationen (z.B. Beratungszentrum für Migranten und Migrantinnen und der ÖIF/Österreichische Integrationsfonds) beobachten die Integrationsverläufe unmittelbar, da sie täglich sowohl mit Afghan/inn/ en zu tun haben als auch mit den entsprechenden rechtlichen und politischen Entwicklungen konfrontiert werden. Die Beratung bezieht sich sowohl auf die Arbeits- und Wohnungssuche als auch auf Ausbildung, Behördenkontakte und andere integrationsrelevante Aspekte. Das Angebot der Organisationen umfasst unter anderem Schulungen, Workshops, Kurse, Einzelberatung, soziale Aktivitäten sowie die Begleitung im Alltag. Zusätzlich wurden direkt aus entsprechenden Behörden wie der Magistratsabteilung 17 - „Integration und Diversität“ Personen, die Integrationsprojekte durchführen, befragt.

Einige Expert/inn/en haben als ehemalige Asylwerber/innen alle Stadien der Integration selbst durchlebt. Um die Perspektive der Afghan/inn/en noch stärker zu 
integrieren, wurden außerdem Vertreter der afghanischen Kultur- und Moscheevereinen interviewt. Diese arbeiten für ein harmonisches Miteinander sowohl innerhalb der afghanischen Community als auch zwischen Afghan/inn/en und Österreicher/inne/n.

Die politische Perspektive, die anhand eines Interviews mit einem zuständigen Landesrat erhoben wurde, zeigt die Bemühungen der Bundesländer und Gemeinden, die Integration zu fördern. Die Unterstützung reicht von der aktiven Aufnahme von Asylwerber/inne/n bis hin zur Begleitung von Asylberechtigten und umfasst alle Lebenslagen einschließlich der sozialen Integration. Auch psychologische Aspekte der Integration und die entsprechenden Herausforderungen wurden in einem Interview analysiert.

Übergeordnetes Ziel der Organisationen und Expert/inn/en ist die Integration der geflüchteten Afghan/inn/en in Österreich, indem Informationen über beide Kulturen angeboten und das Verständnis gefördert wird sowie Unterstützung in allen Lebensbereichen erfolgt. Repräsentant/inn/en der folgenden Institutionen bzw. Organisationen konnten befragt werden:

1. Afghanischer Verein

2. Asylkoordination

3. Aus- und Weiterbildungszentrum (AWZ) Soziales Wien

4. Beratungszentrum für Migranten und Migrantinnen

5. BMEIA

6. Caritas

7. Interface Wien - Core (MA17)

8. Islamische Glaubensgemeinschaft in Österreich, Afghanische Moschee

9. Landesrat Oberösterreich

10. MA 17 - Integration und Diversität

11. Österreichischer Integrationsfond (ÖIF)

12. Universität Wien

Deren Tätigkeitsbereiche umfassen:

1. Beratung (z.B. Arbeits- und Wohnungssuche, Behördengänge, Bildung)

2. Forschung

3. Gerichtliche Gutachten und Übersetzungen bei Asylverfahren o. Ä.

4. Politik

5. Psychotherapeutische Unterstützung

6. Staatliche Integrationsprojekte

7. Vereinsaktivitäten 


\section{Die Fokusgruppendiskussion}

Aus der Kategorie der Expert/inn/en wurden vier aus unterschiedlichen Fachbereichen und mit komplementärem Wissen ausgewählt, um an einer Fokusgruppendiskussion teilzunehmen. Zusätzlich wurden zwei weitere Expert/inn/en eingeladen sowie ein Asylwerber. Insgesamt nahmen also zusätzlich zu vier Personen aus dem Forscher/ innenteam sieben Fachleute an der Diskussion am 19. März 2018 am Institut für Kulturund Sozialanthropologie der Universität Wien teil. Dabei wurde über die ersten Ergebnisse der Studie und daraus abgeleitete relevante Fragestellungen diskutiert. Auf diese Weise wurden zum einen die vorläufigen Erkenntnisse kritisch reflektiert und zusätzliche Informationen eingeholt. Das Spektrum der diskutierten Themen erstreckte sich von Wohnpräferenzen, Spracherwerb, Arbeitsmarkt- und Wohnungsmarktintegration, Wertewandel, Religion und Glaube, über Geschlechterrollen, Demokratie und Rechtsstaat bis zu Asylrecht und Asylverfahren.

\section{Das Interviewer/innenteam}

Das Interviewer/innenteam umfasste zehn Personen und setzte sich sowohl aus Projektmitarbeiter/inne/n sowie neu angeworbenen Interviewer/inne/n zusammen. Bei Letzteren waren muttersprachliche Dari/Farsi-Kenntnisse wichtig, da die wissenschaftlichen Mitarbeiter/innen ausschließlich Befragungen auf Deutsch bzw. Englisch führen konnten. Es wurden gezielt Interviewer/innen eingesetzt, die sprachlich und durch ihre sozialen Netzwerke mehrere ethnische Minoritäten Afghanistans abdeckten (z.B. Hazara, Paschtunen, Tadschiken, Uzbeken). Im Laufe der Erhebung beendeten drei Interviewer/innen ihre Mitarbeit. Bei den muttersprachlichen Interviews gehörten Interviewer/innen und Respondent/inn/en stets demselben Geschlecht an.

Aufgrund der teils fehlenden Ausbildung in qualitativen Forschungsmethoden war eine professionelle Schulung der Interviewer/innen vonnöten, um die Qualität der geführten Interviews zu gewährleisten. Im Vorfeld der Erhebung wurden drei Pretestinterviews durchgeführt und Nachschärfungen beim Fragenkatalog vorgenommen.

\subsection{Erhebungsmethode}

\section{Aufbau von Kontakten zu den Respondent/inn/en}

Die ursprünglich geplante Verwendung des Kontaktdatenpools aus der Pilotstudie (KoHLBACHER et al. 2017) konnte nicht umgesetzt werden, da deren Teilnehmer/innen nicht mehr erreichbar waren oder ein weiteres Interview ablehnten. Um neue Kontakte zu akquirieren, wurden kombinierte Verfahren des Kontaktaufbaus angewendet. Neben den sozialen Netzwerken der afghanischen Interviewer/innen wurden auch Gatekeeper/ innen der afghanischen Community kontaktiert, um Respondent/inn/en zu gewinnen. Diese Methode, ist besonders bei Forschungsvorhaben mit sensiblen Thematiken vorteilhaft (KRUSE 2015). Auf Basis der entstandenen Kontakte wurden mittels eines Schneeballsystems weitere Interviewpartner/innen akquiriert. Ein Vorteil desselben liegt 
darin, dass es sich für schwer erreichbare Personengruppen eignet (JACOB et al. 2011). Konkret bedeutet dies, dass Personen, mit welchen bereits Interviews geführt wurden, um weitere Kontakte gebeten wurden (LEwIS-BEck et al. 2004, p. 1043). Eine Problematik dieser Methode liegt in der Gefahr, selektiv nur bestimmte Gruppen zu erreichen und die Diversität des Feldes nicht erfassen zu können (KRUSE 2015). Dem wurde durch Quotakontrolle entgegengewirkt, d.h., eine breite Verteilung nach ethnischen Herkunftsgruppen, soziodemographischen sowie bildungsbezogenen Variablen wurde angestrebt. Die Projektmitarbeiter/innen begaben sich ins Forschungsfeld und nahmen an Sportveranstaltungen, wie Fußballturnieren, Kulturveranstaltungen von afghanischen Vereinen, Vorträgen etc. teil. So war es möglich, mittels persönlicher Gespräche ein Vertrauensverhältnis aufzubauen.

\section{Die Leitfadeninterviews}

Die Durchführung von leitfadengesteuerten Interviews stellt die Kernmethode der vorliegenden Studie dar. Diese Interviewform zeichnet sich durch einen im Vorhinein erstellten Fragenkatalog aus, der die Grundlage der Erhebung bildet. Um den in qualitativen Interviews erwünschten Erzählfluss der Befragten nicht zu stoppen und längere Erzählphasen anzuregen, ist ein flexibler Umgang mit dem Leitfaden notwendig. Fragen müssen daher an das Gegenüber angepasst und auf etwaige ausgelassene Fragenblöcke zu einem späteren Zeitpunkt zurückgegriffen werden.

\section{Der Leitfaden für die Respondent/inn/eninterviews}

In Orientierung an den Zielen der Studie wurden folgende Teilaspekte als Grundlagen des Fragenkatalogs erfasst:

- Wertvorstellungen,

- Religiosität und religiöse Praxis,

- Demokratieverständnis,

- Einstellungen zur rechtsstaatlichen Ordnung,

- Gender Role Models,

- Einstellungen finanzieller Natur, v.a. zur bedarfsorientierten Mindestsicherung,

- individuelle Integrationsstrategien,

- Ausmaß und Reaktionen auf enttäuschte Erwartungshaltungen,

- soziale Netzwerke,

- Einbindung in Organisationen der afghanischen Community.

Die Erstellung des Leitfadens für die Respondent/inn/eninterviews wurde im Rahmen zahlreicher Meetings des gesamten Projektteams sowie unter Einbeziehung der Rückmeldungen seitens der Repräsentant/innen des Auftraggebers BMEIA erstellt. Sodann erfolgten auf sensible Fragen bezogene Umformulierungen auf Basis von Feedbackschleifen mit Afghan/inn/en. Der in deutscher Sprache erstellte Leitfaden wurde ins Farsi-Dari übersetzt und die Übersetzungen einer Sprachüberprüfung unterworfen. 
Der Leitfaden für die Expert/inn/eninterviews

Der primäre Zweck der Expert/inn/enbefragung lag in einer vertiefenden Analyse, Ergänzung und Reflexion der Resultate aus den Interviews mit den Afghan/inn/en. Die Fachleute sollten ein Feedback liefern und dienten der Erfassung zusätzlicher Meinungsdimensionen sowie der Plausibilitätsprüfung der Hypothesen des Projektteams. Die Schwerpunkte der Befragung waren:

- Erwartungen an und Perspektiven in Österreich: Lebensziele, Bildungsorientierung, Frustrationserfahrungen;

- strukturelle (Bildungssystem, Arbeits- und Wohnungsmarkt) versus soziale Integration (soziale Interaktionsmuster: mit Aufnahmegesellschaft/innerhalb der ethnischen Community, soziale Netzwerke, Vereine der ethnischen Community);

- Einstellungen (Grundwerte, Lebensstile, Religiosität, Geschlechterrollenbilder etc.);

- spezifische Integrationsbarrieren und -probleme;

- Problemkonstellationen in Interaktion mit der österreichischen Aufnahmegesellschaft, Konflikte mit anderen Herkunftsgruppen etc.

Die Interviews wurden in erster Linie muttersprachlich (Farsi-Dari) geführt und auf eine kultursensible Interviewführung Wert gelegt. Etliche der bereits lange in Österreich aufhältigen Afghan/inn/en wünschten in deutscher Sprache befragt zu werden. Konnte der Kontakt zum/r Respondent/in über persönliche Netzwerke hergestellt werden, so konnte eine gewisse Vertrauensbeziehung antizipiert werden. Dies bewirkte, dass auch auf die sensiblen Fragen geantwortet wurde.

Nach der Interviewführung erfolgte die Erstellung eines Protokolls, welches Informationen zum Gesprächsverlauf und der Erzählbereitschaft beinhaltete. Auf Basis der Audioaufnahme wurde eine Transkription in der Interviewsprache durchgeführt und diese dann ins Deutsche übersetzt.

\subsection{Datenauswertung}

Der Gesamtdatenpool umfasste 65 leitfadenorientierte Interviews mit Respondent/ inn/en, 13 Expert/inn/eninterviews sowie die Fokusgruppendiskussion. Die transkribierten Interviews wurden mittels des Analyseprogramms Atlas.ti anhand einer Liste von relevanten Kategorien codiert und die Interviewtranskriptionen einer Inhaltsanalyse unterzogen (vgl. KRUSE 2015). Anschließend wurde das Material auf die Kategorien hin durchsucht und in Auseinandersetzung mit theoretischen Konzepten eine Interpretation der behandelten Themenfelder durchgeführt. 